\title{
Recuperación de Cryptococcus neoformans y C. gattii ambientales y su asociación con aislados clínicos en Cúcuta, Colombia
}

\author{
Asbleide Angarita-Sánchez ${ }^{*} \bowtie \mathbb{E}$ Esp; Denny Cárdenas-Sierra ${ }^{1} \bowtie$ M.Sc; Claudia Parra-Giraldo ${ }^{2} \otimes(\mathbb{P}$ Ph.D; \\ Claudia Diaz-Carvajal ${ }^{1} \otimes$ M.Sc; Patricia Escandon-Hernandez ${ }^{3} \bowtie(\mathbb{M}$ M.Sc. \\ 1 Universidad de Santander, Facultad de Ciencias de la Salud, Grupo BIOGEN, Cúcuta, Colombia. \\ 2 Pontificia Universidad Javeriana, Facultad de Ciencias, Departamento de Microbiología, Unidad de Proteómica y Micosis \\ Humanas, Grupo Enfermedades Infecciosas, Bogotá, Colombia. \\ 3 Instituto Nacional de Salud, Grupo de Microbiología, Bogotá, Colombia. \\ * Correspondencia: as.angarita@udes.edu.co.
}

\section{RESUMEN}

Objetivo. Aislar, identificar y caracterizar molecularmente aislamientos de Cryptococcus patógenos para humanos a partir de muestras ambientales y clínicas de la ciudad de Cúcuta. Materiales y métodos. Se recolectaron 1300 muestras de 446 árboles de 10 especies diferentes, en 10 zonas públicas de Cúcuta. Concomitantemente, se obtuvieron aislados clínicos de Cryptococcus neoformans (junio de 2016-junio de 2017). Se realizó cultivo en agar semillas de Guizottia abysinica, posterior identificación bioquímica y caracterización genética mediante PCR-huella Digital y RFLP-URA5. Resultados. Se determinó prevalencia ambiental para $C$. neoformans de $4.3 \%$ (19 individuos positivos) y $C$. gattii de $0.2 \%$ ( 1 individuo positivo), para un total de 21 aislados y 20 árboles positivos. El parque Santander registró el $47.6 \%$ de la prevalencia global (10/21 aislados), seguido del parque La Victoria con $23.8 \%$ ( $5 / 21$ aislados), correspondientes a C. neoformans. Se obtuvo un aislado de C. gattii en un individuo Ficus benjamina del parque Mercedes Ábrego. El análisis genotípico reveló presencia de C. neoformans var. grubii VNI en el $85.7 \%$ de los aislados ambientales, así como en el $100 \%$ de los clínicos, seguido de VNII y VGII en $9.5 \%$ y $4.8 \%$ de los aislados ambientales, respectivamente. Conclusiones. El muestreo longitudinal de los nichos ambientales previamente reportados del hongo revela su presencia y sugiere que se requiere una vigilancia permanente tanto en el medio ambiente como en los pacientes, especialmente en las zonas endémicas de la ciudad.

Palabras clave: Colombia, criptococosis, genotipo, hábitat, prevalencia (Fuentes: DeCS, MeSH).

\begin{abstract}
Objective. Identify and molecularly characterize Cryptococcus isolates potentially pathogenic to humans from environmental and clinical samples collected in the city of Cúcuta. Materials and methods. We collected 1300 samples from 446 trees belonging to ten different species in ten public spaces in Cúcuta. We also obtained clinical isolates of Cryptococcus neoformans collected between June 2016 and July 2017. Samples were cultivated in Guizottia abysinica seed medium, and then were identified biochemically and characterized by Fingerprinting PCR and RFLP of URA5 gene. Results. C. neoformans displayed an environmental prevalence of $4.3 \%$ (19 positive individuals) and C. gattii $0.2 \%$ ( 1 positive individual); this yields a total of 20 Cryptococcus-positive trees and 21 isolates. The Santander park registered $47.6 \%$ of the global prevalence (10/21 isolates), followed by La Victoria Park with $23.8 \%$ ( $5 / 21$ isolates). One C. gattii isolate was collected from a Ficus benjamina tree located in Mercedes Ábrego park. Genotypic analysis revealed the presence of C. neoformans var. grubii VNI in $85.7 \%$ of environmental isolates as well as $100 \%$ of clinical isolates. VNII and VGII molecular types represented $9.5 \%$ and $4.8 \%$ of environmental isolates respectively. Conclusiones. The longitudinal sampling of previously reported environmental niches of the fungus reveals its presence and suggests that a permanent surveillance in both environment and patients is required, especially in endemic zones of the city.
\end{abstract}

Keywords: Colombia, cryptococcosis, genotype, habitat, prevalence (Sources: DeCS, MeSH).

Como citar (Vancouver)

Angarita-Sánchez A, Cárdenas-Sierra D, Parra-Giraldo C, Diaz-Carvajal C, Escandon-Hernandez P. Recuperación de Cryptococcus neoformans y C. gattii ambientales y su asociación con aislados clínicos en Cúcuta, Colombia. Rev MVZ Cordoba. 2019; 24(1):7137-7144. DOI: https://doi.org/10.21897/rmvz.1601

(C)EI (los) autor (es), Revista MVZ Córdoba 2019. Este artículo se distribuye bajo los términos de la licencia internacional Creative Commons Attribution 4.0 (https://creativecommons.org/licenses/by-sa/4.0/), que permite el uso sin restricciones, la distribución y la reproducción en cualquier medio, siempre que se otorgue el crédito apropiado al autor o autores originales y la fuente. 


\section{INTRODUCCIÓN}

La criptococosis es una micosis que afecta a individuos inmunocompetentes e inmunocomprometidos, especialmente con $\operatorname{SIDA}(\underline{1}, \underline{2}, \underline{3}, \underline{4}, \underline{5}, \underline{6})$. El agente causal de dicha micosis es la levadura encapsulada Cryptococcus spp, con sus dos especies patógenas $C$. neoformans y $C$. gattii. C. neoformans posee a su vez las variedades grubii (correspondiente al serotipo A, patrón molecular VNI y VNII) y la variedad neoformans (correspondiente al serotipo D, patrón VNIV), además del híbrido serotipo AD, patrón molecular VNIII. Por su parte, C. gattii comprende los serotipos B y C (patrón molecular VGI- y VGIV, respectivamente) (7). En 2015 se publicó una revisión de la taxonomía de ambos complejos con una nueva propuesta que incluía siete especies: $C$. neoformans, C. deneoformans, C. gattii, C. bacillisporus, C. deuterogattii, C. tetragattii y C. decagattii (ㅁ).

El estudio de aislados clínicos y ambientales de Cryptococcus como patógeno en humanos es relevante en la región de Cúcuta. La incidencia de la enfermedad en la población general en Colombia fue de 0.23 casos por cada 100.000 personas, siendo Norte de Santander el departamento con mayor incidencia: 0.56 casos por cada 100000 personas. C. neoformans var. grubii patrón molecular VNI se asocia como el principal agente etológico de la criptococosis tanto en individuos inmunocompetentes como inmunodeprimidos. Dada su alta morbi-mortalidad, la patología, a su vez se utiliza como un marcador centinela para monitorear infección por VIH (9). Análisis epidemiológicos y la constante ocurrencia de casos clínicos compatibles con criptococosis muestran a Cúcuta como una ciudad con alta prevalencia de la infección, principalmente aquella causada por Cryptococcus gattii, patrón molecular VGII (10) $)$.

Teniendo en cuenta la asociación del subgrupo genético VGII con alta virulencia y potencial para generar brotes, es importante mantener una vigilancia ambiental, que muestre las concentraciones, y apoyen posibles acciones de prevención $(\underline{10})$. Aunque la infección por C. gattii es minoritaria en Colombia respecto a $C$. neoformans (serotipos $A$ y D), el municipio de Cúcuta, tiene una alta prevalencia $(60 \%)$ en pacientes VIH negativos $(\underline{11})$ y a nivel global se reporta una menor frecuencia de criptococosis por $C$. gattii en pacientes inmunocomprometidos incluyendo VIH/SIDA, los cuales se han asociado a genotipos VGIII y VGIV, como es el caso de California, EUA, considerada zona endémica para VGIII atribuyéndosele infección en un $12 \%$ de éstos (12).

A partir del ambiente, el serotipo $C$. gattii fue aislado por primera vez en Cúcuta en el año 1998(13). Posteriormente, en el año 2011 se obtuvo el primer aislamiento ambiental de C. gattii serotipo B en esta misma ciudad (9).

Dada la alta incidencia de la criptococosis en la ciudad de Cúcuta, el objetivo del presente estudio fue determinar la presencia de $C$. neoformans y $C$. gattii en el área urbana de la ciudad y establecer la asociación de los aislamientos clínicos con aquellos recuperados de muestras ambientales.

\section{MATERIALES Y MÉTODOS}

Sitio de estudio y recolección de muestras. Se recolectaron 1300 muestras de 446 árboles distribuidas así: suelo (442), corteza (434), frutos secos (40) y hojas (384); en el momento de recolección de las muestras se seleccionaban como característica principal la presencia heces de palomas en su superficie. El primer muestreo se realizó en el mes octubre de 2016 en los parques Mercedes Abrego, Simón Bolívar, Arcoíris (La Libertad) y zona periférica al Estadio General Santander. El segundo muestreo se llevó a cabo en el mes de enero del 2017 en los Parques Antonia Santos, La Victoria, Juana Rangel de Cuellar y Parque Santander y el tercero, en el mes de abril del 2017, se realizó en los Parques Nacional y Fuentes de Leones.

Por otra parte, se obtuvieron 6 aislados de $C$. neoformans de líquido cefalorraquídeo (LCR) de pacientes VIH positivos con criptococosis meníngea, procedentes del Hospital Universitario Erasmo Meoz de Cúcuta, entre los meses de junio de 2016 y junio del 2017. Estas muestras correspondieron al $4.3 \%$ de la totalidad de las reportadas a través de la vigilancia pasiva de la criptococosis que se realiza en el país mediante la Encuesta Epidemiológica sobre la Criptococosis en Colombia.

Procesamiento de muestras ambientales. Se realizó un mapeo de los lugares de muestreo donde se identificó a cada individuo (árbol) para la recolección de muestras en cada una de las zonas seleccionadas del área metropolitana de Cúcuta, empleando bolsas plásticas.

Estas fueron procesadas mediante la técnica de extracción de buffer fosfato salino (PBS), suplementando con antibiótico, como se describió previamente $(\underline{14}, \underline{15})$. En breve, $5 \mathrm{~g}$ de muestra se resuspendieron en $25 \mathrm{ml}$ de PBS 1X para homogenización y reposo durante 60 minutos. Posteriormente, se filtró cada homogenizado con gasa estéril, se adicionó el antibiótico y se dejó actuar por 60 minutos.

Un total de $100 \mu$ l de cada preparación fueron sembrados en agar semilla de guizottia para posterior incubación a $27^{\circ} \mathrm{C}$ durante 20 días, con observación periódica semanal, como ha sido referido (16).

Las colonias cremosas, elevadas, con bordes regulares y pigmento café (producción de melanina) obtenidas, fueron observadas al microscopio empleando tinta china (Figura 1). Toda colonia correspondiente a levaduras grandes, redondas y con presencia de cápsula fue sometida a pruebas de degradación de urea (17) y determinación de la especie empleando agar CGB (Agar canavanina glicina azul de bromotimol sódico) (토).

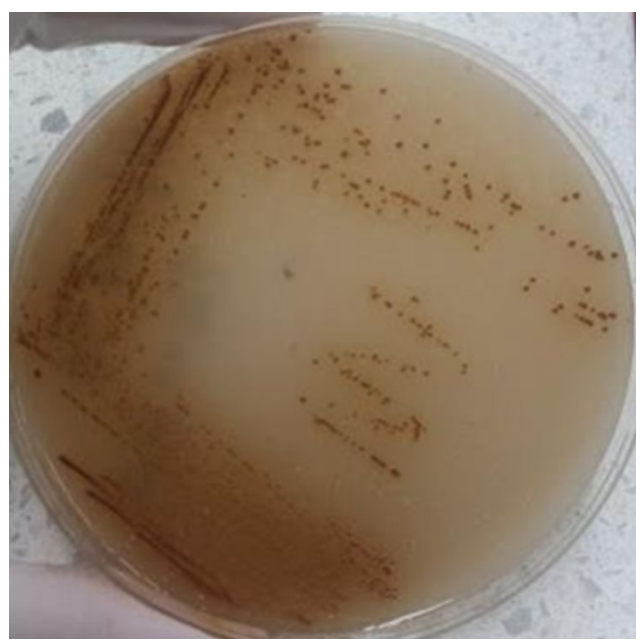

Figura 1. Cryptococcus spp. Agar semilla de guizottia (Producción de melanina). Universidad de Santander, Cúcuta. 
Procesamiento de aislados clínicos. A los aislados de procedencia clínica (Figura 2) igualmente se les realizó cultivo en agar semilla de guizottia, pruebas de degradación de urea (14) y determinación de la especie en agar CGB (Canavanina glicina azul de bromotimol sódico) (18).

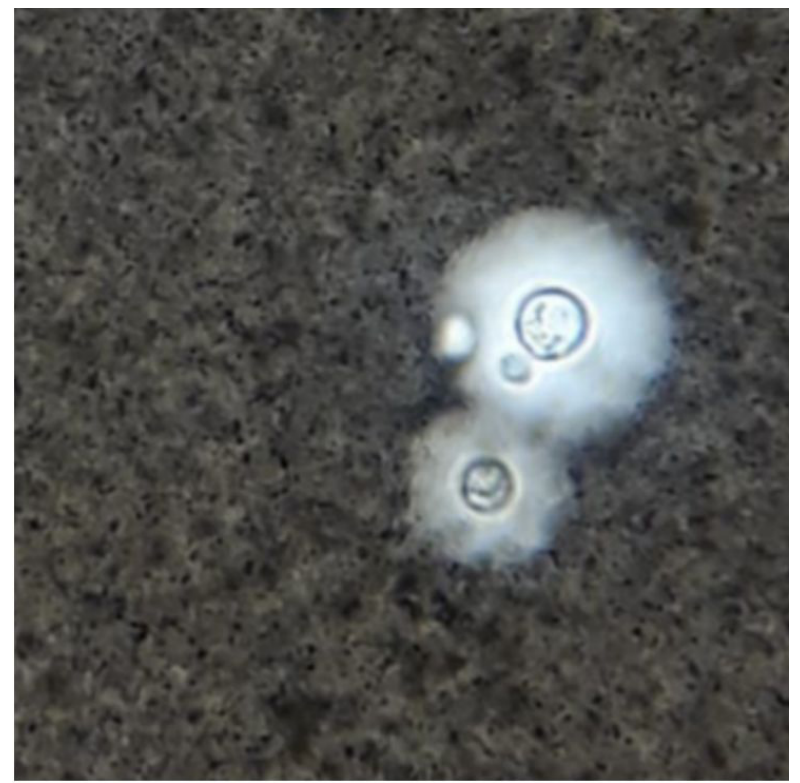

Figura 2. Cryptococcus sp muestra de líquido cefalorraquídeo en tinta china. Grupo de Microbiología, Instituto Nacional de Salud.

Determinación del patrón molecular por PCR-Huella digital. El ADN de alto peso molecular fue extraído, utilizando el método fenol: cloroformo:alcohol isoamílico según Casali et al (19) . Este procedimiento se llevó a cabo utilizando biomasa obtenida de las cepas clínicas y ambientales una vez cultivadas en agar Saboureaud a $27^{\circ} \mathrm{C}$ por 48 horas. Posteriormente se transfirieron colonias del cultivo a tubos de microcentrífuga de 1.5 $\mathrm{ml}$ y se incubaron $\mathrm{a}-20^{\circ} \mathrm{C}$ durante una noche. Después se suspendieron en $500 \mu \mathrm{l}$ de buffer de extracción (Tris $\mathrm{HCl} 50 \mathrm{mM}$, EDTA $50 \mathrm{mM}$, dodecil sulfato sódico al 3\%, 2 - mercaptoetanol 1\%), agitando vigorosamente con vórtex y se incubaron a $65^{\circ} \mathrm{C}$ durante 1 hora. El lisado se extrajo con fenol-cloroformo-alcohol isoamílico (25: 24: $1, \mathrm{vol} / \mathrm{vol} / \mathrm{vol})$. El ADN se recuperó mediante precipitación con isopropanol a $-20^{\circ} \mathrm{C}$ durante una noche, se lavó con etanol al 70\% ( $\mathrm{vol} / \mathrm{vol})$ y finalmente, se resuspendió buffer TRIS - EDTA (TE). La concentración se midió mediante fluorescencia (Quibit 3.0 fluorómetro).

La caracterización molecular de las especies de Cryptococcus spp, se realizó mediante PCR - huella digital, utilizando como cebador único de secuencia especifica microsatélite (GTG) ${ }_{5}$ (5'-GTGGTGGTGGTGGTG-3') descrito por Escandón y colaboradores en 2006 (므).

La mezcla de PCR de $50 \mu \mathrm{L}$ contenía;31.5 $\mu \mathrm{L}$ de agua desionizada estéril, $25 \mathrm{ng}$ de ADN , $5 \mu$ de tampón de PCR 10X (Tris / HCl $10 \mathrm{mM}, \mathrm{pH} 8.3,50 \mathrm{mM} \mathrm{KCl}, 1.5 \mathrm{mM} \mathrm{MgCl}$ ) (Invitrogen, Carlsbad, CA), 0.02 mM de cada dNTP (dATP, dCTP, dGTP y dTTP) (Promega, Madison, WI), acetato de sodio $3 \mathrm{mM}$ (Sigma, Atlanta, GA), $0.8 \mathrm{ng}$ de cebador (para GTG5 la concentración fue $10 \mathrm{ng}$ ), $2 \mathrm{mM} \mathrm{MgCl}, 1$ $\mathrm{ml}$ de albúmina de suero bovino (BSA, $200 \mathrm{mg} \mathrm{mL}^{-1} \backslash$ Delta 1) y 0.05 U Amplitaq. La PCR se realizó durante 35 ciclos en un SimpliAmp ${ }^{\mathrm{TM}}$ Thermal Cycler utilizando, las siguientes condiciones: desnaturalización a $94^{\circ} \mathrm{C}$ durante 20 segundos, hibridación a $50^{\circ} \mathrm{C}$ durante 1 minuto, extensión a $72^{\circ} \mathrm{C}$ durante 20 segundos y un ciclo de extensión final a $72^{\circ} \mathrm{C}$ durante 6 minutos. Los productos de la amplificación, se separaron por electroforesis en un gel de agarosa al $1.4 \%$ en tampón Tris-borato EDTA (TBE) $1 \mathrm{X}$ a $100 \mathrm{~V}$ durante 2 horas.

Los productos de la amplificación $\mathrm{GTG}_{5}$ se tiñeron con Gel red Nucleid Acid Gel Stain Biotium a $0.3 \mathrm{mg} \mathrm{mL}^{-1}$, durante 30 minutos. Las bandas se visualizaron bajo luz UV utilizando marcador de tamaño molecular de $1 \mathrm{~kb}$ en tres pozos para permitir la normalización de los geles. Los tipos moleculares (VNI-VNIV y VGI-VGIV) se asignaron para comparar las cepas de referencia de los ocho tipos moleculares principales cargados en cada gel ( $\underline{21})$.

Determinación del patrón molecular por RFLP- gen URA5 y análisis de asociación. La identificación de los tipos moleculares de Cryptococcus spp., se realizó tanto a las cepas clínicas como ambientales con los dos cebadores URA 5 (5'ATGTCCTCCCAAGCCCTCGACTCCG3') y SJ01 (5'TTAAGACCTCTGAACACCGTACTC3'). La reacción de la PCR se llevó a cabo según lo descrito por Meyer et al (17); los productos de la PCR se sometieron a una doble digestión enzimática con Sau $96 I$ y Hha I a $37^{\circ} \mathrm{C}$ durante 3 horas. Los fragmentos de restricción fueron separados por electroforesis en gel de agarosa al 3\% en tampón Tris-borato EDTA (TBE) $1 \mathrm{X}$ a $100 \mathrm{~V}$ durante 5 horas. Los perfiles de restricción fueron comparados con los patrones obtenidos de las cepas de referencia, utilizando análisis en módulo BioGalaxy del programa Biolomics, versión 3.0.

\section{RESULTADOS}

Caracterización de muestras ambientales. El 75\% de los árboles analizados provenían de 6 parques: parque Simón Bolívar en primer lugar con 80 individuos, seguido de los parques Santander, La Victoria, Nacional, Mercedes Ábrego (con 70, 68, 67 y 50 individuos, respectivamente), siendo esta última cantidad igual para la zona circundante al Estadio General Santander.

A su vez, la diversidad de los individuos analizados en el presente trabajo comprendió 10 especies, siendo el Oití (Licania tomentosa) la más ampliamente distribuida y predominante con el $66.4 \%$ del total de individuos, seguida del Almendro (Terminalia catappa) con 13.2\%, abarcando éstas dos especies aproximadamente el 80 $\%$ de la muestra total. El 20\% restante se distribuyó entre Mamoncillo (Melicoccus bijugatus) $7.2 \%$, Ficus (Ficus benjamina) 5.4\%, Tamarindo (Tamarindus indica) con $2.9 \%$, Chiminango y Totumo (Pithecellobium dulce) $1.8 \%$ cada uno, Samán (Samanea saman), Limón swingle (Swinglea glutinosa) y Mango (Mangifera indica), $0.4 \%$ cada uno.

El cultivo inicial reveló la presencia de 21 aislados compatibles con Cryptococcus sp, 20 de los cuales correspondían a C. neoformans (95.2\%) y 1 a C. gattii $(4.8 \%)$, obtenidos a partir de 20 de los 446 individuos, ya que en un mismo árbol se obtuvo doble positividad para C. neoformans (Tabla 1). Por lo anterior, se obtuvieron prevalencias de $4.3 \%$ y $0.2 \%$, para $C$. neoformans y $C$. gattii, respectivamente. 
Tabla 1. Descripción de muestras positivas para C.neoformans y C.gattii

\begin{tabular}{|c|c|c|c|c|c|c|}
\hline $\begin{array}{c}\text { Identificación de } \\
\text { muestra }\end{array}$ & $\begin{array}{l}\text { Tipo de } \\
\text { muestra }\end{array}$ & Zona de procedencia & Especie del individuo & $\begin{array}{l}\text { Especie de } \\
\text { Cryptococcus }\end{array}$ & Variedad & $\begin{array}{c}\text { Genotipo } \\
\text { Cryptococcus }\end{array}$ \\
\hline 25 & Corteza & Parque Santander & Mango (Mangifera indica) & C. neoformans & grubii & VN I \\
\hline 35 & Corteza & Parque Santander & Oití (Licania tomentosa) & C. neoformans & grubii & VN I \\
\hline 37 & Tierra & Parque Santander & Oití (Licania tomentosa) & C. neoformans & grubii & VN I \\
\hline $39 a *$ & Hojas & Parque Santander & Oití (Licania tomentosa) & C. neoformans & grubii & VN I \\
\hline $39 b *$ & Tierra & Parque Santander & Oití (Licania tomentosa) & C. neoformans & grubii & VN I \\
\hline 47 & Corteza & Parque Santander & Mango (Mangifera indica) & C. neoformans & grubii & VN I \\
\hline 58 & Tierra & Parque Santander & Mango (Mangifera indica) & C. neoformans & grubii & VN I \\
\hline 63 & Corteza & Parque Santander & Samán (Samanea saman) & C. neoformans & grubii & VN I \\
\hline 68 & Corteza & Parque Santander & Oití (Licania tomentosa) & C. neoformans & grubii & VN I \\
\hline 69 & Tierra & Parque Santander & Tamarindo (Tamarindus indica) & C. neoformans & grubii & VN I \\
\hline 1 & Corteza & Parque La Victoria & Totumo (Crescentia cujete) & C. neoformans & grubii & VN I \\
\hline 10 & Tierra & Parque La Victoria & Mango (Mangifera indica) & C. neoformans & grubii & VN II \\
\hline 44 & Corteza & Parque La Victoria & Chiminango (Pithecellobium dulce) & C. neoformans & grubii & VN II \\
\hline 45 & Corteza & Parque La Victoria & Limón swingle (Swinglea glutinosa) & C. neoformans & grubii & VN I \\
\hline 49 & Corteza & Parque La Victoria & Mamoncillo (Melicoccus bijugatus) & C. neoformans & grubii & VN I \\
\hline 2 & Tierra & Parque Mercedes Ábrego & Ficus (Ficus benjamina) & C. gattii & gattii & VG II \\
\hline 5 & Corteza & Fuente de Leones & Oití (Licania tomentosa) & C. neoformans & grubii & VN I \\
\hline 7 & Tierra & Parque Antonia Santos & Oití (Licania tomentosa) & C. neoformans & grubii & VN I \\
\hline 12 & Corteza & Parque Arcoíris-La Libertad & Almendro (Terminalia catappa) & C. neoformans & grubii & VN I \\
\hline 32 & Tierra & Estadio General Santander & Oití (Licania tomentosa) & C. neoformans & grubii & VN I \\
\hline 58 & Corteza & Parque Simón Bolívar & Almendro (Terminalia catappa) & C. neoformans & grubii & VN I \\
\hline
\end{tabular}

*Muestras derivadas del mismo individuo

Aislados ambientales con su zona de procedencia, especie del individuo y aislamiento con su tipo molecular identificado por PCR- huella digital con cebador (GTG) 5 y por RFLP gen URA5.

En total, el parque Santander ocupó el primer lugar en prevalencia con $47.6 \%$ de los 21 aislados obtenidos, seguido del parque La Victoria $(23.8 \%)$ y finalmente los parques Fuente de Leones, Antonia Santos, Arcoíris (La Libertad), Simón Bolívar y alrededores del Estadio General Santander (cada uno con $4.8 \%$ de los aislados), exclusívamente para $C$. neoformans var grubii, ya que un único aislado de $C$. gattii se obtuvo en el parque Mercedes Ábrego ( $4.8 \%$ de los aislados).

A su vez, el cálculo de la prevalencia por cada zona pública estudiada reveló datos sobresalientes especialmente para dos de éstas: parque Santander con $12.8 \%(9 / 70$ individuos fueron positivos) y parque La Victoria con $7.3 \%$ (5/68 individuos fueron positivos), en ambos casos sólo para C. neoformans. Se muestra la prevalencia individual para las distintas zonas evaluadas (Figura 3).

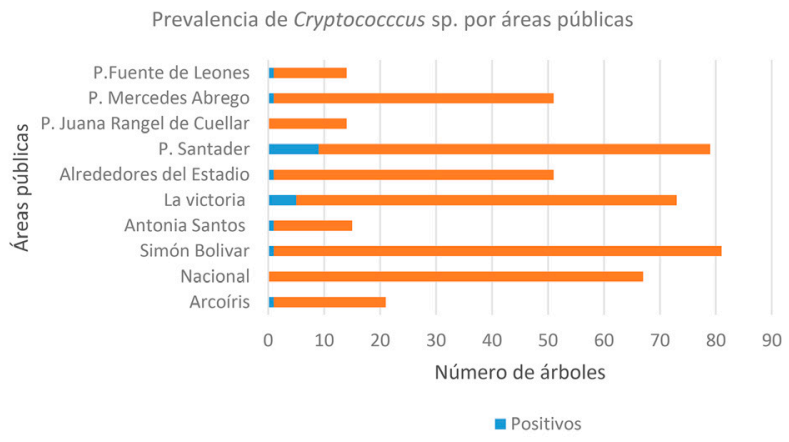

Figura 3. Prevalencia para Cryptococcus sp en áreas públicas de Cúcuta.
El análisis de positividad según tipo de muestra evidenció un predominio de aislados de $C$. neoformans obtenidos a partir de corteza y suelo con el 60\% (12/20 aislados) y $35 \%$ ( $7 / 20$ aislados), respectivamente, mientras que sólo uno fue obtenido a partir de hojas (5\%). Lo anterior implica una prevalencia general para $C$. neoformans a partir de corteza, suelo y hojas, de $3.0 \%$ (de 434 muestras), $1.6 \%$ (de 442 muestras) y $0.3 \%$ (de 384 muestras), respectivamente.

Respecto a $C$. gattii, se obtuvo única positividad en una muestra de suelo (Tabla 1). En general, no se obtuvieron aislados a partir de las 40 muestras de frutos secos analizados en el presente estudio.

La presencia de $C$. neoformans VNI se concentró en un $55.5 \%$ en el parque Santander (10/18 aislados), seguido de un $16.6 \%$ en parque la Victoria (3/18 aislados) y una minoría se distribuyó en los parques Fuente de Leones, Antonia Santos, Arcoíris (La Libertad), Simón Bolívar y alrededores del Estadio General Santander, con 5.3\% de los aislados para cada zona (1/18 aislados), mientras que el tipo molecular VNII se halló exclusivamente en el parque la Victoria, aislado a partir de suelo y corteza de las especies arbóreas Mango (Mangifera indica) y Chiminango (Pithecellobium dulce), respectivamente.

Por otra parte, el aislado de C. gattii correspondió a VGII, siendo a su vez el único obtenido a partir del parque Mercedes Abrego, a partir de suelo asociado a un individuo de Ficus (Ficus benjamina) (Tabla 1).

Caracterización de muestras clínicas. El análisis genotípico a partir de muestras clínicas reveló la presencia de $C$. neoformans en todos los casos, correspondientes en su totalidad al tipo molecular VNI (Figura 4). 

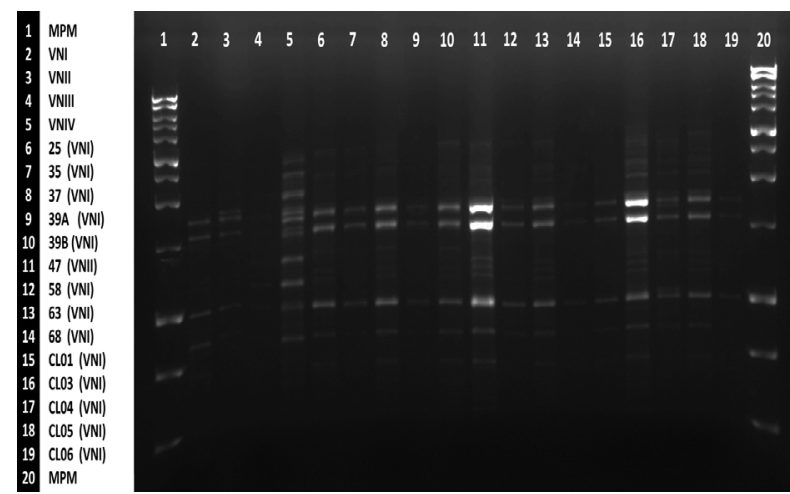

Figura 4. PCR huella digital de aislados clínicos y ambientales de Cryptococcus neoformans var. grubii usando el cebador (GTG), con marcador de peso molecular $1 \mathrm{~kb}$ y cepas de control.

Relación genética entre los aislados de $C$. neoformans de procedencia clínica y ambiental. La PCR huella digital y el RFLP del gen URA5, reveló que el patrón genético encontrado en esos aislamientos de pacientes también está presente en la mayoría de los aislamientos ambientales. Se observó una correlación entre estos dos tipos de aislamientos a nivel genético /parque Santander (10/18 aislamientos), seguido por $16.6 \%$ en Parque la Victoria ( $3 / 18$ aislamientos), y una minoría se distribuyó entre la Fuente de Leones, Antonia Los parques Santos, Arcoiris (La Libertad) y Simón Bolívar y los alrededores del Estadio General Santander, con el $5.3 \%$ de los aislamientos para cada zona (1/18 aislamientos); el tipo molecular VNII se encontró exclusivamente en Victoria Park en el suelo y la corteza de los árboles Mango (Mangifera indica) y Chiminango (Pithecellobium dulce), respectivamente.

Por el contrario, el aislamiento de $C$. gattii correspondió a VGII, siendo a su vez el único obtenido de Mercedes Abrego Park de un Ficus individual (Ficus benjamina) (Tabla 1).

\section{DISCUSIÓN}

En el contexto ecológico se ha referido predominio de $C$. neoformans, especialmente $C$. neoformans var grubii VNI y VNII (serotipo A), cuyo hábitat se extiende también a excretas de aves (22). Estos dos tipos moleculares son igualmente los más prevalentes en casos de criptococosis $(97 \%)$ en individuos inmunosuprimidos en Colombia $(21,22,23)$ y otros países incluso europeos (24). Sin embargo, la presencia ambiental de C. gattii reviste especial relevancia en torno a su capacidad de generar enfermedad en individuos inmunocompetentes y su potencial de adaptación a distintas condiciones geográficas, dejando de ser exclusivo de zonas tropicales y subtropicales, para pasar a hallarse también en zonas templadas y lluviosas (므).

El presente trabajo abarcó 10 zonas públicas altamente concurridas de la ciudad de Cúcuta, en las cuales en su mayoría se reveló la presencia de patógenos del género Cryptococcus sp, exceptuando los parques Nacional y Juana Rangel de Cuéllar. La prevalencia hallada fue consistente con lo referido previamente
(22), mayor en general para $C$. neoformans (4.3\%) respecto a $C$. gattii $(0.2 \%)$ a razón de $21.5: 1$, pero por otra parte, contrastante con datos previos reportados para la ciudad de Cúcuta en particular (años 2008 y 2009), habiéndose referido como de $0.07 \%$ de manera similar para ambas especies del hongo, incluso con un tamizaje significativamente mayor de individuos (3634) procedentes de zonas La Libertad, San Eduardo y alrededores del Estadio General Santander (26).

Según nuestros hallazgos, la positividad para $C$. neoformans mostró predominio en el parque Santander (47.6\% de los 21 aislados), seguido del parque La Victoria $(23.8 \%)$ y finalmente los parques Fuente de Leones, Antonia Santos, Arcoíris (La Libertad), Simón Bolívar y alrededores del Estadio General Santander (cada uno con $4.8 \%$ de los aislados), exclusívamente para $C$. neoformans variedad grubii. Tomando en cuenta lo anterior, podemos concluir que los parques Santander (especialmente y con prevalencia individual superior al $10 \%$ ) y La Victoria, constituyen nichos ecológicos relevantes para $C$. neoformans, aportando éste último además variabilidad genética al hallarse tanto el tipo molecular VNI como el VNII (única zona con hallazgo de éste último).

Se contó con una variedad de especies arbóreas que evidenciaron ser nichos del hongo, especialmente Oití (Licania tomentosa), ampliamente distribuida en las zonas evaluadas, a partir de la cual se identificó el $38 \%$ del total de aislados ( $8 / 21$ aislados), todos correspondientes a $C$. neoformans VNI, además de hallarse en diversidad de fuentes (corteza, tierra y hojas), seguida del Mango (Mangifera indica), a partir del cual se obtuvieron 4 aislados (19\%), tanto en tierra como en corteza, correspondientes a $C$. neoformans variedad grubbii (VNI y VNII) y el Almendro (Terminalia catappa), con dos aislados (9.5\%), identificados como C. neoformans VNI, en contraste con el reporte previo de éste último como hábitat tanto de $C$. neoformans como de C. gattii (25).

En el parque Santander de manera interesante, los cinco aislados obtenidos de la especie arbórea oití (Licania tomentosa) correspondieron a cuatro individuos, puesto que uno de ellos fue doble positivo, revelando la presencia de $C$. neoformans VNI simultáneamente en suelo o tierra adyacente al mismo y en sus hojas.

Este caso único de doble positividad para Cryptococcus neoformans, registrado en el presente trabajo no tiene antecedente en la ciudad, en lo que respecta al tipo de árbol (Licania tomentosa).

Según lo publicado por Firacative et al $(\underline{26})$, el rastreo de más de 4300 muestras procedentes de árboles de diversas zonas de Cúcuta y áreas cercanas a la vivienda de pacientes infectados por C. gattii serotipo B (VGI/a), arrojó sólo un aislamiento de éste patógeno, dos aislamientos de $C$. gattii serotipo C (VGIII/a) y tres de C. neoformans serotipo A (VNI/a), donde uno de éstos últimos, junto con el único aislado de C. gattii (VGI/a) se obtuvieron de un mismo árbol pero en ese caso de especie Ficus (Ficus benjamina) en el sector San Eduardo.

De igual manera, en los alrededores del Estadio General Santander, también en suelo de un mismo árbol Ficus 
(Ficus benjamina) se hallaron simultáneamente $C$. gattii (VGIII/a) y C.neoformans serotipo A (VNI/a) ( $\underline{26})$.

Con relación a lo anterior, el presente trabajo reveló positividad para ésta última especie de árbol, pero correspondiente al aislamiento de $C$. gattii VGII a partir de muestra de tierra en el parque Mercedes Ábrego (sin antecedente), en plena zona céntrica de la ciudad de Cúcuta. ésta fue la cuarta zona con mayor cantidad de individuos tamizados, sin embargo, sólo uno de sus 50 árboles resultó positivo, tratándose de un individuo Ficus (Ficus benjamina).

Ficus benjamina alberga tanto a C. gattii como a C. neoformans (26), se reviste de importancia si se considera la presencia de los genotipos VGI, VGII y VGIII tanto en muestras ambientales como en pacientes con enfermedad criptocóccica $(10,21,23)$. Estos tres tipos moleculares también han sido asociados a casos de criptococosis humana y animal en el occidente de Estados Unidos. Particularmente los genotipos VGII y VGIII de origen ambiental se han identificado como el posible agente etiológico de la micosis en individuos VIH positivo (27).

Los hallazgos en la zona circundante al Estadio General Santander son consistentes con un reporte previo, el cual mostró la presencia de C. neoformans (26), permitiendo concluir sobre su persistencia en el área. Sin embargo, esta positividad se observó por primera vez en árboles de la especie Licania tomentosa, reflejando una variación tanto de los tipos de hábitat del hongo, como de las especies del patógeno que circulan en una zona determinada.

Dada la amplia distribución ambiental de C. neoformans y C. gattii, y su asociación a patología en individuos inmunosuprimidos e incluso inmunocompetentes, resulta relevante el rastreo permanente de zonas comunes de

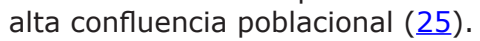

En concordancia con lo anterior, el reconocimiento de la identidad entre aislados de Cryptococcus sp de procedencia clínica respecto a la ambiental, mediante la comparación de genotipos circulantes es de gran interés teniendo en cuenta además el aumento de los casos de criptococosis en individuos VIH positivos, al comparar los lapsos de tiempo 1997-2005 con el periodo 20062010 (3000 casos/millón de habitantes y 3300 casos/ millón de habitantes, respectivamente) $(\underline{21}, \underline{23})$. A lo anterior se suma que la incidencia de criptococosis afecta también a menores de edad en Colombia (menores de 16 años), donde la infección por VIH constituye un factor de riesgo reconocible en apenas la mitad de los casos, quedando por factores de riesgo concomitantes. Resulta preocupante, sin embargo, que esta incidencia es siete veces mayor para el mismo grupo poblacional en Norte de Santander ( 0.122 casos/100.000 habitantes), respecto a la media nacional (0.017 casos/100.000 habitantes) (28).

El análisis genotípico de las especies patógenas procedentes de 6 muestras clínicas en el presente trabajo reveló exclusivamente la presencia de $C$. neoformans var grubii VNI ( $100 \%$ de los casos). en consistencia con el amplio predominio registrado para éste en estudios

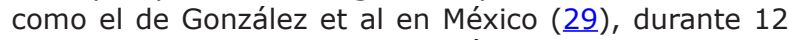
años, a partir de 166 muestras clínicas (no se especifica si expresamente de pacientes $\mathrm{VIH}+$ ), quienes refirieron prevalencia para $C$. neoformans de $92.2 \%$, respecto a C. gattii $(7.8 \%)$ y correspondientes a VNI $(74.6 \%)$, VNII $(9 \%)$, VNIII $(4.8 \%)$, VNIV $(3.6 \%)$, VGI $(3.6 \%)$, VGII

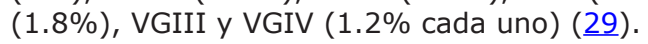

Es necesario ampliar el número de aislamientos clínicos que permitan determinar asociaciones con los aislamientos ambientales del patrón VNII, así como $C$. gattii presente en el ambiente.

Es de resaltar que Cúcuta se caracteriza por sectores ampliamente arborizados y la presencia de gran cantidad de aves destacándose principalmente la Paloma de la especie Columbia livia en los parques estudiados, siendo más representativa la presencia de estas en el Parque Santander en donde este trabajo permite confirmar el nicho de la levadura, otros estudios también evidencia la relación existente entre los nichos ecológicos y estas aves $(\underline{30})$; de ahí la importancia de una vigilancia epidemiológica debido a la cercanía con los humanos y el hecho de que pueden trasladarse de un parque al otro.

Estos datos relacionados con la alta prevalencia de pacientes con diagnóstico de criptococosis y su relación genética con los aislados ambientales es de gran aporte en los programas de salud pública.

\section{Conflictos de interés}

Los autores informan no tener ningún conflicto de interés.

\section{Agradecimientos}

Al Grupo de Microbiología Instituto Nacional de Salud, a la Unidad de Investigación en Proteómica y Vicerrectoría de investigación Proyecto ID 8247 y a la Universidad de Santander, financiación de proyecto número PICF0215385771745EJ. A los auxiliares de investigación estudiantes del Programa de Bacteriología y Laboratorio Clínico Universidad de Santander campus Cúcuta: Erika Y Guerrero Martínez Kelly, J Carrascal Quintero, Eliana J Moreno Mora, Damarys L Gelves Trujillo, Aymet P Rubio Cano, Gyovany A Rincón Castrillón. 


\section{REFERENCIAS}

1. Ye F, Xie J-x, Zeng Q-s, Chen G-q, Zhong S-q, Zhong $\mathrm{N}-\mathrm{s}$. Retrospective analysis of 76 immunocompetent patients with primary pulmonary cryptococcosis. Lung. 2012; 190(3):339-346. DOI: https://doi. org/10.1007/s00408-011-9362-8 PMid:22246551

2. Lin X, Heitman J. The biology of the Cryptococcus neoformans species complex. Annu Rev Microbiol. 2006; 60:69-105. DOI: https://doi.org/10.1146/ annurev.micro.60.080805.142102 PMid:16704346

3. Perfect JR. Management of cryptococcosis: how are we doing? PLoS med. 2007; 4(2):e47. DOI: https://doi.org/10.1371/journal.pmed.0040047 PMid: 17284156

4. Perfect JR, Dismukes WE, Dromer F, Goldman DL, Graybill JR, Hamill RJ, et al. Clinical practice guidelines for the management of cryptococcal disease: 2010 update by the Infectious Diseases Society of America. Clin Infect Dis. 2010; 50(3):291322. DOI: https://doi.org/10.1086/649858 PMid: 20047480

5. Alvarez-Moreno CA, Cortes JA, Denning DW. Burden of Fungal Infections in Colombia. J Fungi. 2018; 4(2):41. DOI: https://doi.org/10.3390/jof4020041 PMid:29561795 PMCid:PMC6023354

6. Esher SK, Zaragoza O, Alspaugh JA. Cryptococcal pathogenic mechanisms: a dangerous trip from the environment to the brain. Mem Inst Oswaldo Cruz. 2018; 113(7):1-15. DOI: https://doi. org/10.1590/0074-02760180057 PMid:29668825

7. Kwon-Chung $\mathrm{KJ}$, Fraser JA, Doering $T L$, Wang ZA, Janbon G, Idnurm A, et al. Cryptococcus neoformans and Cryptococcus gattii, the etiologic agents of cryptococcosis. Cold Spring Harb Perspect Med. 2014; 4(7):a019760. DOI: https://doi. org/10.1101/cshperspect.a019760 PMid:24985132

8. Hagen F, Khayhan K, Theelen B, Kolecka A, Polacheck I, Sionov E, et al. Recognition of seven species in the Cryptococcus gattii/Cryptococcus neoformans species complex. Fungal Genet Biol. 2015; 78:16-48. DOI: https://doi.org/10.1016/j. fgb.2015.02.009 PMid:25721988

9. Escandón P, Lizarazo J, Agudelo CI, Casta-eda E. Cryptococcosis in Colombia: Compilation and Analysis of Data from Laboratory-Based Surveillance. J Fungi. 2018; 4(1):32. DOI: https:// doi.org/10.3390/jof4010032 PMid:29494502

10. Lizarazo J, Escandón P, Agudelo CI, Firacative C, Meyer W, Casta-eda E. Retrospective study of the epidemiology and clinical manifestations of Cryptococcus gattii infections in Colombia from 1997-2011. PLoS Negl Trop Dis. 2014; 8(11):e3272. DOI: https://doi.org/10.1371/ journal.pntd.0003272 PMid:25411779
11. Lizarazo J, Chaves O, Pe-a Y, Escandón P, Agudelo CI, Casta-eda E. Comparación de los hallazgos clínicos y de supervivencia entre pacientes VIH positivos y VIH negativos con criptococosis meníngea en un hospital de tercer nivel. Acta Med Colomb. 2012; 37(2):49-61.

12. Chen SC-A, Meyer W, Sorrell TC. Cryptococcus gattii infections. Clin Microbiol Rev. 2014; 27(4):9801024. DOI: https://doi.org/10.1128/CMR.0012613 PMid:25278580

13. Callejas A, Ordonez N, Rodriguez M, Castaneda E. First isolation of Cryptococcus neoformans var. gattii, serotype $C$, from the environment in Colombia. Med Mycol. 1998; 36(5):341-344. DOI: https://doi.org/10.1080/02681219880000531 PMid: 10075505

14. Paliwal D, Randhawa H. Evaluation of a simplified Guizotia abyssinica seed medium for differentiation of Cryptococcus neoformans. J Clin Microbiol. 1978; 7(4):346-348. PMid:357441

15. Escandón P, Quintero E, Granados D, Huérfano S, Ruiz A, Casta-eda E. Isolation of Cryptococcus gattii serotype $B$ from detritus of Eucalyptus trees in Colombia. Biomédica. 2005; 25(3):390-397. DOI: https://doi.org/10.7705/biomedica.v25i3.1363 PMid: 16276686

16. Staib F, Seibold M, Antweiler E, Fröhlich B. Staib Agar Supplemented with a Triple Antibiotic Combination for the Detection of Cryptococcus neoformtans in Clinical Specimens: Staib-Agar mit einer Dreifach-Antibiotika-Kombination für den Nachweis von Cryptococcus neoformans in klinischem Untersuchungsmaterial. Mycoses. 1989; 32(9):448-454. DOI: https://doi. org/10.1111/j.1439-0507.1989.tb02280.x PMid:2691887

17. Kwon-Chung K, Wickes BL, Booth J, Vishniac HS, Bennett JE. Urease inhibition by EDTA in the two varieties of Cryptococcus neoformans. Infect Immun. 1987; 55(8):1751-1754. PMid:3112009

18. Min K, Kwon-Chung K. The biochemical basis for the distinction between the two Cryptococcus neoformans varieties with CGB medium. Zentralbl Bakteriol Mikrobiol Hyg A. 1986; 261(4):471480. DOI: https://doi.org/10.1016/S0176$\underline{6724(86) 80079-7}$

19. Casali AK, Goulart L, Rosa e Silva LK, Ribeiro ÂM, Amaral AA, Alves $\mathrm{SH}$, et al. Molecular typing of clinical and environmental Cryptococcus neoformans isolates in the Brazilian state Rio Grande do Sul. FEMS Yeast Res. 2003; 3(4):405-415. DOI: https:// doi.org/10.1016/S1567-1356(03)00038-2 
20. Escandón P, Sánchez A, Martínez M, Meyer W, Casta-eda E. Molecular epidemiology of clinical and environmental isolates of the Cryptococcus neoformans species complex reveals a high genetic diversity and the presence of the molecular type VGII mating type a in Colombia. FEMS Yeast Res. 2006; 6(4):625-635. DOI: https://doi.org/10.1111/ j.1567-1364.2006.00055.x PMid:16696659

21. Escandón $\mathrm{P}$, de Bedout C, Lizarazo J, Agudelo CI, Tobón Á, Bello S, et al. Cryptococcosis in Colombia: Results of the national surveillance program for the years 2006-2010. Biomédica. 2012; 32(3):386398. DOI: https://doi.org/10.7705/biomedica. v32i3.707 PMid:23715187

22. Chowdhary A, Randhawa HS, Prakash A, Meis JF. Environmental prevalence of Cryptococcus neoformans and Cryptococcus gattii in India: an update. Crit Rev Microbiol. 2012; 38(1):1-16. DOI: https://doi.org/10.3109/1040841X.2011.606426 PMid:22133016

23. Lizarazo J, Linares $M$, de Bedout $C$, Restrepo Á, Agudelo CI, Casta-eda E. Estudio clínico y epidemiológico de la criptococosis en Colombia: resultados de nueve a-os de la encuesta nacional, 1997-2005. Biomédica. 2007; 27(1):94-109. DOI: https://doi.org/10.7705/biomedica.v27i1.236 PMid: 17546227

24. Cogliati M, Zamfirova RR, Tortorano AM, Viviani MA, Network FC. Molecular epidemiology of Italian clinical Cryptococcus neoformans var. grubii isolates. Sabouraudia. 2013; 51(5):499-506. DOI: https://doi.org/10.3109/13693786.2012.751642 PMid:23286351

25. Mak S, Vélez N, Casta-eda E, Escandón P. The fungus among us: Cryptococcus neoformans and Cryptococcus gattii ecological modeling for Colombia. Journal of Fungi. 2015; 1(3):332344. DOI: https://doi.org/10.3390/jof1030332 PMid:29376914 PMCid:PMC5753128
26. Firacative C, Torres G, Rodríguez MC, Escandón P. First environmental isolation of Cryptococcus gattii serotype $B$, from Cúcuta, Colombia. Biomedica. 2011; 31(1):118-123. DOI: https://doi. org/10.7705/biomedica.v31i1.342 PMid:22159490

27. Springer DJ, Billmyre RB, Filler EE, Voelz K, Pursall $R$, Mieczkowski PA, et al. Cryptococcus gattii VGIII isolates causing infections in HIV/AIDS patients in Southern California: identification of the local environmental source as arboreal. PLoS Pathog. 2014; 10(8):1-19. DOI: https://doi.org/10.1371/ journal.ppat.1004285 PMid:25144534

28. Lizarazo J, Escandón P, Agudelo CI, Casta-eda E. Cryptococcosis in Colombian children and literature review. Mem Inst Oswaldo Cruz. 2014; 109(6):797804. DOI: https://doi.org/10.1590/00740276130537 PMid:25317708

29. González GM, Casillas-Vega N, Garza-González E, Hernández-Bello R, Rivera G, Rodríguez JA, et al. Molecular typing of clinical isolates of Cryptococcus neoformans/Cryptococcus gattii species complex from Northeast Mexico. Folia Microbiol. 2016; 61(1):51-56. DOI: https://doi.org/10.1007/ s12223-015-0409-8 PMid:26109075

30. Montagna MT, De Donno A, Caggiano G, Serio F, De Giglio O, Bagordo F, et al. Molecular characterization of Cryptococcus neoformans and Cryptococcus gattii from environmental sources and genetic comparison with clinical isolates in Apulia, Italy. Environ Res. 2018; 160:347-352. DOI: https://doi. org/10.1016/j.envres.2017.09.032 PMid:29054089 